\title{
RIGHT VENTRICULAR PRESSURE CURVES IN CONGENITAL AND ACQUIRED HEART DISEASE
}

\author{
BY
R. SHANAHAN,* G. S. MYERS, E. DEL CAMPO, A. I. FRIEDLICH, AND G. SCANNELL \\ From Massachusetts General Hospital, Boston, U.S.A.
}

Received June 3, 1958

The shape of the right ventricular pressure curves, other than those found in constrictive pericarditis and right ventricular failure, have received little attention partly due to a tendency to ascribe variations to artefacts rather than to genuine differences. Harris (1955) called attention to the characteristic patterns found in normal persons, patients with pulmonary hypertension, and those with pulmonary stenosis. The present communication attempts to study the problem in more detail than the works of either Harris (1955) or Luisada and Liu (1956). The findings are based on a study of the records obtained by the cardiac catheterization unit of the Massachusetts General Hospital, Boston, and especially on the tracings obtained from 79 patients with mitral valve disease, 20 with pulmonary disease, and 66 with various congenital heart defects.

Technique. The patients were given mild barbiturate sedation and a prophylactic injection of an antibiotic (usually penicillin), before the start of the studies. The zero level was taken as $5 \mathrm{~cm}$. below the sternal angle, and pressures were recorded through number 6 or 7 Cournand catheters, connected to a Sanborn capacitance electromanometer. Simultaneous pressure and electrocardiographic tracings were recorded on a Sanborn Polyviso electrocardiograph.

Contours of the Right Ventricular Pressure Curve. The basic patterns are as follows.

Type A: The dicrotic curve. The normal right ventricular pressure curve shows a rapid ascent to the maximum pressure produced by systole. Opening of the pulmonary valve (taken as the approximate level at which the right ventricular pressure exceeds the diastolic pressure in the pulmonary artery), produces only a slight slurring on the upstroke of the curve, and does not alter its contour significantly. The pressure in the right ventricle falls rapidly after the peak value has been reached, but the downstroke of the curve shows a secondary rise of pressure, thereby producing a dicrotic notch. This secondary upstroke is found also in the tracings obtained from the pulmonary arteries of these patients, and therefore occurs when the right ventricle and the pulmonary artery are still a common chamber. The descent from this secondary wave is also slurred at the approximate level of the pulmonary arterial diastolic pressure, and after this, the pressure continues to fall smoothly until the pressure in the right ventricle falls slightly below its presystolic level. The remainder of the diastolic portion of the curve shows a gradual ascent to the beginning of the atrial systolic pressure wave, but a dip and plateau effect does not occur. Although this type of curve is not often found in the presence of severe disease involving the lesser circulation, occasional exceptions were found in this series.

Type B: The anacrotic curve. The diastolic portion of this curve is similar to that found in the dicrotic curve, but the systolic portion is different. The upstroke shows an abrupt change in the direction of inscription of the curve, sometimes marked by a notch, and the summit of the curve occurs much later than in the preceding type. This pattern was found in patients with a high pulmonary arteriolar resistance, in some instances of severe mitral valve disease with normal pulmonary arteriolar resistances, and in certain patients with infundibular stenosis.

* Work undertaken while on an Ainsworth Scholarship, granted by University College, Cork. Present address: St. Stephen's Hospital, Cork. 
Type C: The systolic plateau curve of mild pulmonary hypertension. In these patients the right ventricular pressure rises rapidly to a maximum, but does not fall immediately, as in the dicrotic curve, with the result that the pressure is maintained at a plateau before the period of rapid descent begins. The remainder of the curve is similar to the corresponding portions of the other two curves, already described. This pattern was found in cases in which the severity of the disease was intermediate between the two preceding groups. Examples of this type of curve were found in patients with mitral valve disease, and also in patients with chronic pulmonary disease.

Type D: The high pressure systolic plateau curve. This curve has a contour that differs from that of the preceding type, in that, while each has a systolic plateau, followed by a rapid descent to right ventricular diastolic levels, the right ventricular systolic pressure is much higher in this than in the type $C$ curve. In addition, this type of curve usually shows a distinct notch at the beginning of the plateau. Examples of this pattern were found only in patients with either Fallot's tetralogy or large ventricular septal defects.

Type E: The symmetrical peaked curve. This is characterized by a much slower ascent than is found in the other curves. After the peak pressure has been reached, the pressure falls at a rate approximately equal to that of the systolic ascent, with the result that an isosceles triangle effect is produced. This pattern is

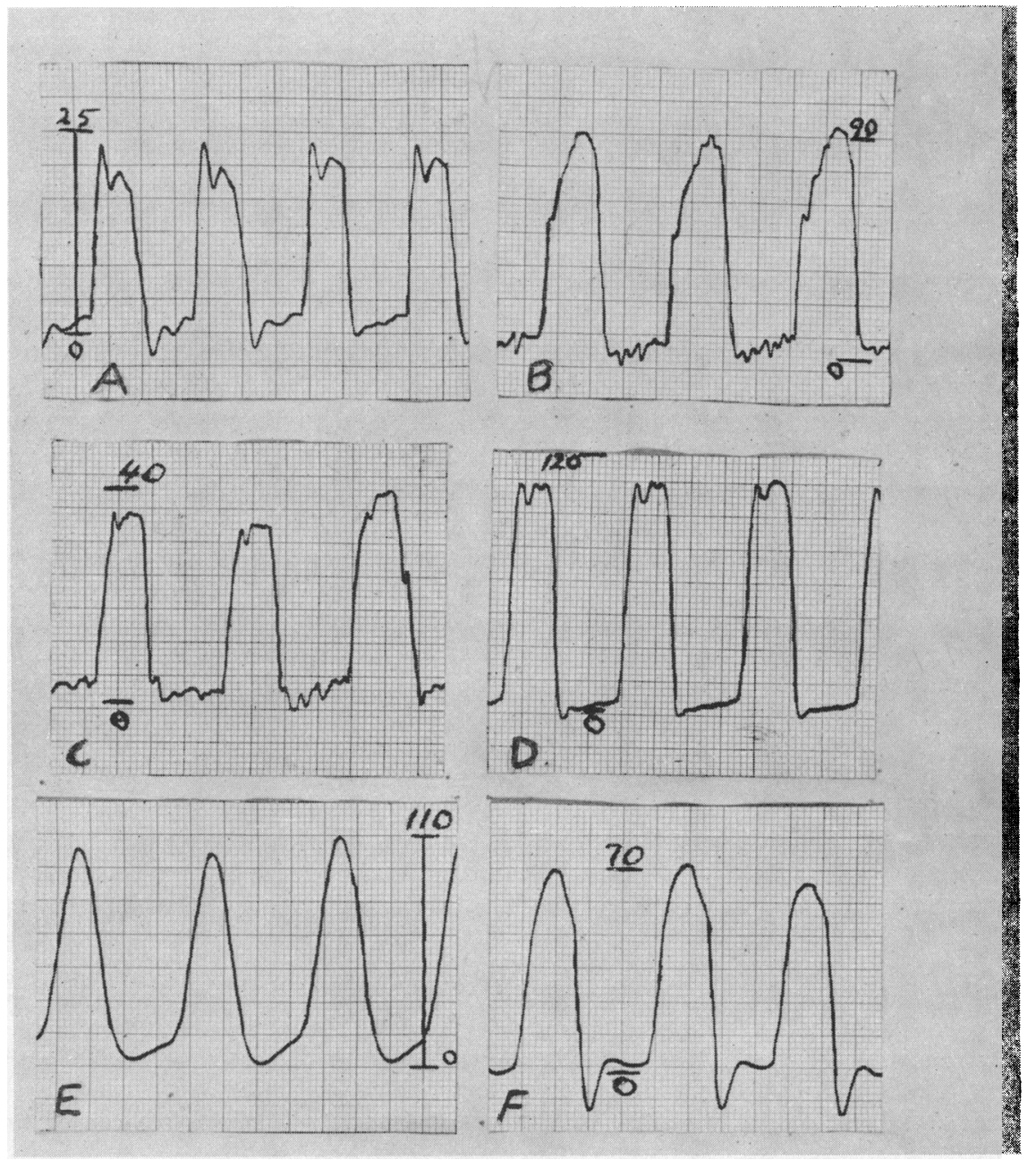

FIG. 1.-(A) dicrotic curve; (B) anacrotic curve; (C) systolic plateau curve of mild pulmonary hypertension; (D) high pressure systolic plateau curve; (E) symmetrical peaked curve; (F) anacrotic curve, with diastolic dip and plateau. 
highly suggestive of pulmonary valvular stenosis (with intact ventricular septum). However, premature beats that fail to open the pulmonary valve produce a similarly shaped curve, even in the absence of cardiac abnormality.

The diastolic dip and plateau. The features of this pattern have been fully described by Hansen et al. (1951). The phenomenon may be associated with either a dicrotic or an anacrotic systolic curve, but in every instance in which it was found thus far severe heart disease was present. Consequently, it was found more useful to include all tracings with a diastolic dip and plateau in a single group, rather than to classify these patients on the basis of the systolic portion of the curve alone.

Almost all of the tracings examined could be classified into one of the foregoing groups, if the entire tracing was studied. However, certain types of artefacts are common and are frequently found in published reports. The most common form is that produced by damping, which is especially apt to occur at the end of a length of tracing. Overshooting is also common, especially if the volume output of the right ventricle is increased. Frequently damping is not sufficiently severe to obscure the pattern of the tracing, but in certain instances it may do so.

The use of different sensitivities in the recording of tracings may also be a source of some confusion. The right ventricular pressure curve of patients with thyrotoxicosis is, at first sight, very similar to one of pulmonary stenosis, but the pressure values are very different, being approximately normal in the former, but raised in the latter. The curve found in thyrotoxicosis is really a dicrotic one, but the downstroke may be so rapid that the dicrotic notch is not as conspicuous as normally, with the result that the descent appears almost smooth.

\section{The Right Ventricular Curve in Mitral Valve Disease}

The significance of the different right ventricular patterns was illustrated when the records of patients with mitral valve disease were studied. Post-operative studies were excluded, and this left 84 tracings available for this investigation, of which only 5 could not be classified on account of artefact. Operative findings were available in 62 of the remaining 79 patients.

In 15 instances, type A (dicrotic) curves were found. In 11 of these 15, operation was not advised, as the final assessment of the patient was that the degree of mitral valve disease was only minor, the calculated mitral valve area (Gorlin and Gorlin, 1951) exceeding $1.7 \mathrm{sq} . \mathrm{cm}$. in all, while the right ventricular systolic pressures ranged between 20 and $48 \mathrm{~mm}$. $\mathrm{Hg}$. In all of these 11 patients, the pulmonary arteriolar resistances were within normal limits. The remaining 4 patients with dicrotic curves were explored surgically. In one, the mitral valve was reported to be "only very moderately stenosed"; in the second the surgeon found "a moderate stenosis and slight regurgitation"; "moderate stenosis and moderate regurgitation" were present at operation on the third patient; and in the last a tight stenosis was found, in association with a right ventricular systolic pressure of $48 / 0$ and a calculated mitral valve area of $1.0 \mathrm{sq} . \mathrm{cm}$. In these 4 operated cases, the calculated pulmonary arteriolar resistances were also found to be within normal limits.

There were 45 patients with type B (anacrotic) curves and of these 39 had operations. In 37 instances the surgeon found a tight mitral stenosis, associated with slight mitral regurgitation in 6 patients. The 2 remaining had larger appertures with considerable mitral regurgitation. Of the 6 unoperated patients with anacrotic curves, medical treatment was considered sufficient for 3 , while surgery was not advised for the remaining 3, owing to the patient's age, the likelihood of predominant mitral regurgitation, or the patient's terminal status. The operated patients were divided into those in whom the right ventricular systolic pressure exceeded $50 \mathrm{~mm}$. $\mathrm{Hg}$ ( 25 patients), and those in whom it was less than that figure (14 patients). Although the degree of mitral valve involvement tended to be somewhat more severe in the first, i.e. those with the higher right ventricular pressures, than in the second group, the height of the right ventricular systolic pressure was found to correlate much better with the pulmonary arteriolar resistance (Wood, 1954). Only 4 of the 25 patients in the first group had pulmonary arteriolar resistances less than 5 units, whereas 11 of the 14 in the second group had normal pulmonary resistances.

Type $C$ curves (systolic plateau curve of mild pulmonary hypertension), were found in 5 of the 79 patients with mitral disease in this series, and in 4 of the 5 instances operation was performed. In one the right ventricular systolic pressure was $55 \mathrm{~mm}$. $\mathrm{Hg}$, and the calculated mitral valve area was $1.0 \mathrm{sq} . \mathrm{cm}$.: at operation, a tight mitral stenosis was found. In the remaining 4 the right ventricular systolic pressures were less than $50 \mathrm{~mm}$. $\mathrm{Hg}$, and the calculated mitral valve areas varied between 1.1 and 1.4 sq. $\mathrm{cm}$. Three of these were operated upon, and mitral regurgitation was present in addition to moderate mitral stenosis. The patient with the calculated valve area of $1.4 \mathrm{sq} . \mathrm{cm}$. was not operated upon. The pulmonary arteriolar resistances in all these five were normal. 
Regardless of the configuration of the systolic portion of the right ventricular pressure curve, all patients showing a diastolic dip and plateau pattern were found at operation to have severe mitral stenosis. Therefore, the remaining 14 cases with mitral disease who exhibited this pattern are considered in this special category, irrespective of the systolic portion of their curves. Five of this group had dicrotic systolic curves, and the other 9 had anacrotic curves. The patients with elevated pulmonary arteriolar resistances all had anacrotic right ventricular systolic curves, but while some of those with normal pulmonary resistances had dicrotic curves, others had anacrotic ones.

None of the patients with mitral disease had either Type D or Type E curves.

TABLE I

\begin{tabular}{|c|c|c|c|c|c|c|}
\hline \multirow[t]{2}{*}{ Type of curve } & \multirow[t]{2}{*}{ No. } & \multirow{2}{*}{$\begin{array}{l}\text { No. operated } \\
\text { upon }\end{array}$} & \multicolumn{2}{|c|}{ Operative findings } & \multicolumn{2}{|c|}{ Assessment of unoperated cases } \\
\hline & & & $\begin{array}{c}\text { Severe } \\
\text { M.S. or M.R. }\end{array}$ & Mild M.S. & $\begin{array}{c}\text { Severe } \\
\text { M.S. or M.R. }\end{array}$ & Mild M.S. \\
\hline $\begin{array}{c}\text { A } \\
\text { B } \\
\text { C } \\
\text { Diastolic dip } \\
\text { and plateau }\end{array}$ & $\begin{array}{r}15 \\
45 \\
5 \\
14\end{array}$ & $\begin{array}{r}4 \\
39 \\
4 \\
14\end{array}$ & $\begin{array}{r}1 \\
39 \\
1 \\
14\end{array}$ & $\begin{array}{l}3 \\
0 \\
3 \\
0\end{array}$ & $\begin{array}{l}0 \\
3 \\
0 \\
0\end{array}$ & $\begin{array}{r}11 \\
3 \\
1 \\
0\end{array}$ \\
\hline
\end{tabular}

Note: M.S. $=$ mitral stenosis; M.R. $=$ mitral regurgitation.

Summary. The figures show a correlation between the contour of the right ventricular curve and the degree of involvement of the mitral valve. The dicrotic curve was usually found in association with mild or moderately severe mitral disease and a normal pulmonary arteriolar resistance. The type $\mathrm{C}$ curve (i.e. the systolic plateau of mild pulmonary hypertension) generally occurred in somewhat more severe cases. When the contour of the systolic curve was anacrotic or when a diastolic dip and plateau was present, the mitral valve was always severely involved.* This proved true even when the right ventricular pressure was not strikingly raised (i.e. less than $50 \mathrm{~mm}$. $\mathrm{Hg}$ ), the height of the right ventricular systolic pressure being related more to the pulmonary arteriolar resistance than to the size of the mitral orifice.

\section{The Right Ventricular Curve in Pulmonary Disease}

Twenty patients with a variety of pulmonary diseases, including emphysema, pneumoconiosis, pulmonary fibrosis, and byssinosis, were studied.

Dicrotic right ventricular systolic pressure curves were found in 6 of the patients. In 4 of these the right ventricular pressure was between 28 and $40 \mathrm{~mm}$. $\mathrm{Hg}$, and the pulmonary arteriolar resistance in each instance was within normal limits. The fifth had a right ventricular systolic pressure of $60 \mathrm{~mm}$. $\mathrm{Hg}$, despite a normal pulmonary arteriolar resistance, due to a cardiac output elevated to $8 \cdot 1 \mathrm{l} / \mathrm{min}$. The sixth had a right ventricular pressure of $75 \mathrm{~mm}$. $\mathrm{Hg}$, and an elevated pulmonary arteriolar resistance of 20 units. The stroke output was only $30 \mathrm{ml}$., which was felt to be an important factor in the production of a dicrotic curve in this patient.

Six of these 20 patients had anacrotic curves, and with one exception, they had high right ventricular systolic pressures and elevated pulmonary arteriolar resistances. The one exception was in a patient with fibrosis of the lungs, who had a normal right ventricular pressure, normal pulmonary arteriolar resistance, and a normal stroke output. In 5 patients the right ventricular curve was of the type $C$ variety: in all of these, the right ventricular pressures were at the upper limit of normal, and the pulmonary arteriolar resistances were either normal or only moderately elevated.

Summary. In general a dicrotic curve occurred in those cases with a normal pulmonary resistance, an anacrotic contour in those with high resistances, and a type $\mathrm{C}$ curve in those with only moderately high resistances. A dicrotic pattern occurred in one patient with a high pulmonary resistance and a very small stroke output. The possible significance of this will be considered later.

* Since these figures were compiled, a patient with mild mitral stenosis (valve area $=1.8 \mathrm{sq}$. $\mathrm{cm}$.) was found to have a diastolic dip and plateau, showing that the rule is not absolute. 


\section{The Right Ventricular Curve in Congenital Heart Disease}

The right ventricular pressure curves displayed striking variations in the cases of congenital heart disease.

Ventricular Septal Defect. The tracings from 16 patients with isolated ventricular septal defects and from 2 with ventricular septal defects, infundibular stenosis, and left-to-right shunts were available for study. Thirteen of the 16 patients with isolated ventricular septal defects had elevated pulmonary arteriolar resistances, and in each instance, the right ventricular systolic curve was anacrotic in type. In 4 of these 13, a diastolic dip and plateau was also present in the tracing. The two with normal pulmonary arteriolar resistances were found to have dicrotic right ventricular pressure curves: in each of these, a substantial leftto-right shunt was present. The last patient in this group had a high pressure systolic plateau curve (type D): the resistance could not be measured as he was only an infant. The 2 patients with ventricular septal defects and associated infundibular stenosis offer an interesting comparison. The right ventricular systolic pressures were approximately equal $(90$ and $85 \mathrm{~mm}$. $\mathrm{Hg}$ ), as were the mean pulmonary arterial pressures $(28$ and $20 \mathrm{~mm}$.), but the shunts were of very different magnitudes, and the right ventricular pressure curves reflected this difference. The patient with the larger shunt (pulmonary flow three times the systemic flow) had an anacrotic curve, whereas the other (pulmonary flow 1.6 times the systemic flow) had a high pressure systolic plateau curve.

Atrial Septal Defect. 18 patients with isolated atrial septal defects were analysed. The 6 with dicrotic curves had normal pulmonary arteriolar resistances, whereas the 7 with raised resistances were found to have anacrotic curves. The remaining 5 tracings could not be classified because of artefacts. This high incidence of artefacts was related to the very high flow rates through the right ventricle in these cases.

The Tetralogy of Fallot. The right ventricular pressure curves in patients with the tetralogy of Fallot were different from those found in pure pulmonary stenosis. This may be of considerable assistance in differentiating Fallot's tetralogy from pulmonary stenosis with intact ventricular septum and right-to-left shunt through an atrial defect. Eight patients with Fallot's tetralogy were examined, the diagnosis being confirmed at operation in three and at autopsy in a fourth. All showed a high pressure systolic plateau curve. This type of curve was found in only two other instances: an infant with a large ventricular septal defect only and another patient with a ventricular septal defect and infundibular stenosis.

Pure Pulmonary Stenosis. Wiggers (1928) was the first to call attention to the fact that under experimental conditions, pulmonary stenosis alters the appearance of the right ventricular systolic pressure curve to a single monophasic hump. More recently, Harris (1955) emphasized this fact, and suggested that infundibular stenosis produces a different pattern, i.e. one similar to that found in pulmonary hypertension, and described above as the anacrotic curve. Twenty-two records were available for this study, when patients with infundibular stenosis and those with pulmonary valvular stenosis combined with septal defects (but excluding cyanotic tetralogies) were included with those of pure (isolated) pulmonary valvular stenosis. The characteristic type of curve (i.e. the symmetrical peaked curve) was found in 11 cases of pulmonary stenosis, including 2 with associated atrial septal defects. In 2 cases of pulmonary stenosis with only moderate elevation of the right ventricular systolic pressure (about $60 \mathrm{~mm}$. Hg.) dicrotic curves occurred. Four of the 22 had associated ventricular septal defects; in 3 of these anacrotic curves occurred, while the fourth had a high pressure systolic plateau curve. One patient with infundibular stenosis, proved by catheterization and later by operation, had a symmetrical peaked curve. Two of the others without ventricular septal defects were found to have anacrotic curves, and in one of these the pull-back tracing was suggestive but not diagnostic of infundibular stenosis. The remaining two tracings could not be classified.

The findings therefore support the view that the symmetrical peaked curve, associated with much right ventricular hypertension, strongly suggests pulmonary stenosis, usually valvular. An anacrotic curve in patients with pulmonary stenosis suggests an associated ventricular septal defect or infundibular rather than valvular narrowing. A dicrotic curve may be found in patients with mild pulmonary stenosis.

\section{Discussion}

In view of the general agreement between the contour of the right ventricular systolic pressure curve and the degree, and sometimes the nature, of the various lesions studied, it is of interest to speculate on the possible factors that may be responsible for alteration in shape of these curves.

The delayed rise of pressure during the descent from the peak of the dicrotic curve occurs about 0.08 sec. after the initial peak, and it is suggested here that the secondary pressure wave is a 
phenomenon produced by the rebound of the primary wave from the pulmonary vascular bed. If this theory be true, the initial peak of the dicrotic curve is the point where the right ventricle is contracting to maximum effect, and the pressure produced by it should begin to fall immediately afterwards. Reduction of pressure will be augmented if the volume of blood in the common chamber of the right ventricle and pulmonary arteries also decreases; but this reduction of volume cannot occur as efficiently as normally if the pulmonary arteriolar resistance is increased or if there is mitral valve disease or left ventricular failure. In these cases, the secondary wave tends to be superimposed at a higher level of the downstroke of the right ventricular curve, and therefore tends to produce the systolic plateau of the type $\mathrm{C}$ curve. If the resistance to the primary wave is still greater, the rebound would (other factors being equal) also increase in magnitude and consequently the anacrotic pattern would be produced. The notch on the upstroke of the anacrotic curve is therefore equivalent to the peak of the dicrotic curve, whereas the apex of the systolic portion of the anacrotic tracing is the counterpart of the secondary pressure wave on the downstroke of the dicrotic curve.

Since a key factor in the production of the anacrotic curve is the inability of a sufficient volume of blood to escape from the pulmonary artery, the importance of stroke output is obvious. The only occasion that a dicrotic curve was found in the presence of a much raised pulmonary arteriolar resistance was in a patient with a very small stroke output. The anacrotic curve was often found in patients with normal pulmonary resistances, if tight mitral stenosis or severe mitral regurgitation was present, thereby showing that the obstruction to flow, necessary for the production of this pattern, could occur beyond the small vessels in the lungs.

The systolic plateau, found in the tracings of patients with the tetralogy of Fallot, would appear to be produced in a different manner to the somewhat similar plateau of the type $\mathrm{C}$ curve. Owing to the overriding aorta, the right ventricular pressure in these cases cannot exceed the aortic and the left ventricular systolic pressure, but it is also true that it cannot fall if the left ventricular pressure is maintained. The right ventricular curve therefore shows a prolonged systolic elevation followed by an abrupt drop, i.e. a pattern similar to that usually found in the left ventricular tracing. This explanation could also account for the occurrence of this type of curve in certain cases of ventricular septal defects in whom the shunt is from left to right.

The simplest contour is the symmetrical peaked curve of pulmonary stenosis. This is similar to the curve produced, at a lower pressure level, by an ectopic beat that fails to open the pulmonary valve. The feature common to these two conditions, is the failure of the pulmonary artery and the right ventricle to become a common chamber. Consequently, the work of the right ventricle is performed against a fairly constant resistance, and in addition, the right ventricular curve is protected against rebound pressure waves from the pulmonary vascular bed.

\section{SUMMARY}

Five basic types of right ventricular systolic pressure curves are described in addition to the diastolic "dip and plateau". The relationship between these patterns and various cardiac conditions is examined and theories are proposed to account for the various systolic patterns.

\section{REFERENCES}

Gorlin, I. R., and Gorlin, S. G. (1951). Amer. Heart J., 41, 1.

Hansen, A. T., Eskildsen, P., and Goetzsche, H. (1951). Circulation, 3, 881

Harris, P. (1955). Brit. Heart J., 17, 173.

Luisada, A. A., and Chi Kong Liu (1956). Cardiac Pressures and Pulses. Grune \& Stratton Publications, Wiggers, C. J. (1928). The Pressure Pulses in the Cardiovascular System. Longmans, Green \& Co., London. Wood, P. (1954). Brit. med. J., 1, 1051. 